\title{
El proceso de investigación de los estudiantes de la Feria Nacional de Ciencias e Ingeniería de Coahuila
}

\section{The research process of the students of the National Science and Engineering Fair of Coahuila}

\author{
MUÑOZ-LÓPEZ, Temístocles $\dagger^{*}$, MARTÍNEZ-SOSA, Vanessa, RAMOS-JAUBERT, Rocío Isabel y \\ ESPERICUETA-MEDINA, Marta Nieves
}

Universidad Autónoma de Coahuila, Facultad de Ciencia, Educación y Humanidades

ID $1^{\text {er }}$ Author: Temístocles, Muñoz-Lopez / ORC ID: 0000-0003-4940-5730, Researcher ID Tomson: T-7834-2018, CVU CONACYT ID: 202437

ID $1^{\text {er }}$ Coautor: Vanessa, Martínez-Sosa / ORC ID: 0000-0001-9442-8363, CVU CONACYT ID: 333696

ID $2^{\text {do }}$ Coautor: Rocío Isabel, Ramos-Jaubert / ORC ID: 0000-0003-3289-5390, Researcher ID Tomson: T-1652-2018

ID $3^{\text {er }}$ Coautor: Marta Nieves, Espericueta-Medina / ORC ID: 0000-0002-4924-4332, Resercher ID Thomsom: T-15002018, arXiv Author ID: Espericueta2018. CVU CONACYT ID: 372705

DOI: $10.35429 / J E S C .2020 .11 .4 .19 .32$

Recibido: 10 de Marzo, 2020; Aceptado 30 de Junio, 2020

Resumen

Se desarrolló una investigación con el objetivo de elucidar los procesos de investigación qué siguen los estudiantes en diferentes niveles educativos para desarrollar proyectos de ingeniería dentro de la Feria Nacional de Ciencias e Ingenierías de Coahuila, con la base de datos que utiliza el Consejo Estatal de Ciencia y Tecnología de Coahuila (COECyT) que consta de 135 proyectos de los que se obtuvieron 311 casos, evaluados por 62 investigadores expertos de designados por el COECyT. Dado que los formatos de evaluación estaban en escalas tipo Likert, se transformaron a escala estandarizada centesimal y se analizaron con estadísticos de tendencia central, de dispersión, correlación y análisis discriminante. La conclusión indica que la elaboración de proyectos estudiantiles tiene procesos originales que no se sujetan a una metodología estricta, antes bien, los estudiantes desarrollan sus proyectos con ayuda de los docentes proponiendo y desarrollando soluciones paso a paso, en cada etapa de la investigación, excepto en los requisitos protocolarios del concurso. También podemos afirmar que los proyectos tienen una identidad metodológica en promedio del $50 \%$ con su nivel educativo, de acuerdo con el análisis discriminante.

Proceso de investigación, Proyecto de ingeniería, Escalas, Niveles educativos

\begin{abstract}
An investigation was developed with the aim of elucidating the research processes that students follow at different educational levels to develop engineering projects within the Feria Nacional de Ciencias e Ingenierías de Coahuila, with the database used by the Consejo Estatal de Ciencia y Tecnología de Coahuila (COECyT), which consists of 135 projects from which 311 cases were obtained, evaluated by 62 expert researchers designated by the COECyT. Given that the evaluation formats were in Likert-type scales, they were transformed to a standardized centesimal scale and analyzed with statistics of central tendency, dispersion, correlation and discriminant analysis. The conclusion indicates that the development of student projects has original processes that are not subject to a strict methodology, rather, students develop their projects with the help of teachers proposing and developing solutions step by step, in each stage of the investigation, except in the formal requirements of the contest. We can also affirm that the projects have an average methodological identity of $50 \%$ in each educational level, according to the discriminant analysis.
\end{abstract}

Research process, Engineering project, Scales, Educational levels

Citación: MUÑOZ-LÓPEZ, Temístocles, MARTÍNEZ-SOSA, Vanessa, RAMOS-JAUBERT, Rocío Isabel y ESPERICUETA-MEDINA, Marta Nieves. El proceso de investigación de los estudiantes de la Feria Nacional de Ciencias e Ingeniería de Coahuila. Revista Ciencias de la Educación. 2020. 4-11: 19-32

\footnotetext{
* Correspondencia del Autor (Correo electrónico: temistoclesmunoz@uadec.edu.mx)

$\dagger$ Investigador contribuyendo como primer autor.
} 


\section{Introducción}

Entendiendo la epistemología como el estudio crítico a posteriori que analiza los métodos, técnicas e instrumentos que un investigador pone en juego para encontrar la verdad o resolver un problema, resulta interesante encontrar los patrones de desarrollo de la investigación que los estudiantes sin experiencia en el desarrollo de procesos científicos, Utilizan en la práctica para resolver problemas de diversa índole, que implican desarrollos de ingeniería y de ciencia pura o básica. En esta práctica de la investigación es común encontrar comunidades epistémicas que tienen y aceptan sus métodos de investigación, de acuerdo con la práctica que realizan. según Ignacio Méndez (Méndez Ramírez, 2011) la investigación es un proceso complejo que requiere una cuidadosa consideración de sus objetivos para determinar el marco de referencias y su diseño, de acuerdo con los recursos de que se dispone, y en ello se advierten los principales aspectos metodológicos y cuidados necesarios para concluir con éxito cualquier estudio. Esto es lo que se pretende elucidar en la Feria Nacional de la Ciencia y la Investigación que se realiza anualmente en los diferentes estados de la República, considerando proyectos desarrollados por estudiantes desde Secundaria hasta Educación Superior.

Trabajando en convenio, el Consejo Nacional de Ciencia y Tecnología (CONACYT) y el Consejo Estatal de Ciencia y Tecnología de Coahuila (COECyT) con fundamento en el Programa Especial de Ciencia, Tecnología e Innovación (PECITI) 2014- 2018, y con el objeto de impulsar la investigación científica y tecnológica entre las y los jóvenes en los diferentes sistemas educativos de la entidad, así como fomentar las vocaciones científicas, convocan anualmente a estudiantes coahuilenses interesadas e interesados en el desarrollo de proyectos científicos, tecnológicos y de innovación a participar en la Feria Nacional de Ciencias e Ingenierías, Fase Coahuila (COECyT, 2018).

En lo anterior podemos indicar que las variantes y énfasis de distintas etapas del proceso investigativo depende en mucho de los fines y los recursos que se involucran o están disponibles.
Así, podemos afirmar que hay tres Clases de investigación desde las perspectivas teleológica y operacional, sin que esto pretenda formar una tipología, sino más bien una caracterización de los métodos más comunes de las diferentes comunidades epistémicas, que derivarán posteriormente en protocolos. Por ejemplo, tenemos las investigaciones descriptivas y explicativas que tienden a promover el desarrollo científico y/o tecnológico y la Innovación (RD\&I: Research, Development and Innovation). Estas tres clases son:

1. Investigación científica tradicional. Es la investigación más rigurosa y con un formato general. que puede ser abordada por un método inductivo como en la investigación original, la generación de paradigmas o las de tesis doctorales. Es más común realizarla por un método hipotético-deductivo como en la mayoría de las investigaciones de las ciencias duras.

2. Investigación protocolaria. Es una investigación desarrollada bajo las normas metodológicas, procedimientos, instrumentos y técnicas estrictas que define una comunidad epistémica o profesional de acuerdo con sus propios criterios profesionales. Por ejemplo, los protocolos de la investigación jurídica, o la investigación médica. El texto del Dr. Ignacio Ramírez Méndez contiene ocho formas de protocolo de los cuales se describen su estructura, utilidad y características, y se señalan sus ventajas y desventajas.

3. Investigación para aplicación y desarrollo tecnológico. Es la investigación orientada específicamente a resolver problemas productivos o sociales buscando la innovación, más que a generar conocimiento o desarrollar teoría. Por ejemplo, la desarrollada en la Feria Nacional de la Ciencia. 
En este contexto, la presente investigación realizada con la base de datos de la FENACI 2018 tiene como interrogante de investigación ¿cuál es el proceso de investigación que siguen los noveles estudiantes investigadores en cada nivel académico de Secundaria, Preparatoria (Nivel Medio Superior) y Educación Superior de acuerdo con los proyectos Tecnológicos que presentan en la Feria Nacional de Ciencia e Investigación en Coahuila?

\section{Objetivo}

El objetivo de la presente investigación fue elucidar los procesos de investigación qué siguen los estudiantes en diferentes niveles educativos para desarrollar proyectos de ingeniería que implican innovación y creatividad en la obtención de un producto tecnológico.

\section{Perspectiva teórica}

En la presente década se ha insistido en que México es un país alejado de la ciencia y la tecnología. Esto implica no sólo subdesarrollo, sino también una percepción nacional acerca de que la ciencia y la tecnología no forman parte de la cultura y, aunque en el discurso se resalte su potencial para el desarrollo nacional, en una visión íntima de la sociedad mexicana, se piensa que la inversión en estos campos resulta infructuosa. Se ha insistido en que, desde la secundaria, donde hay materias específicas de ciencias, la situación no es muy diferente.

La visión de ciencia y de enseñanza de la ciencia apegadas al dictado y la memoria dan una imagen distorsionada de la misma $y$, efectivamente, marginal e inútil. En su libro, Fernando Flores compila los trabajos de varios autores que describen la problemática que tienen los profesores en torno a las concepciones de la naturaleza de la ciencia y los conocimientos de las disciplinas que imparten, así como los problemas de la formación docente en este campo, también observan las características que deben cumplir los materiales educativos para la enseñanza de las ciencias, José Antonio Chamizo expone los análisis educativos sobre los trabajos prácticos en la enseñanza de las ciencias y da cuenta del insuficiente impacto que los esfuerzos de las instituciones investigadoras de los problemas de la enseñanza de las ciencias han tenido en las políticas educativas nacionales (Flores-Camacho, 2012).
Algunos autores buscan ayudar a construir los conocimientos básicos para diseñar y realizar una investigación con técnicas cualitativas o cuantitativas de manera rigurosa y adecuada a los objetivos y el contexto de una investigación en desarrollo. Insisten en que cuando el análisis es tan solo una de las etapas de la rigidez metodológica, pone los métodos por encima de los objetivos de la investigación. Así, la explicación de la metodología cuantitativa está adaptada a situaciones habituales en proyectos y procesos de desarrollo, y surge en gran medida de nuestra experiencia práctica como facilitadoras, investigadores y docentes. La metodología se trata desde una perspectiva epistemología realista, en la que la combinación de técnicas cualitativas y cualitativas no se considera problemática, sino que resulta esencial para acercarnos a la realidad al unir extensión y profundidad. (Hueso \& Cascant, 2012)

Los autores que prefieren métodos mixtos (MM) combinan la perspectiva cuantitativa (cuanti) y cualitativa (cuali) en un mismo estudio, con el objetivo de darle profundidad al análisis cuando las preguntas (interrogantes) de investigación son complejas. Así, se han presentado cuatro modelos de MM más utilizados en estudios relacionados con las ciencias de la salud en las últimas dos décadas, estos son: 1) modelo para desarrollar instrumentos; 2) modelo explicativo o aclaratorio; 3) modelo de triangulación; y 4) modelo de transformación longitudinal, así como algunos lineamientos para su utilización en la investigación educativa. Así como los MM le ofrecen al investigador la oportunidad de descubrir orientaciones novedosas, ensayar con variadas estrategias y encontrar sentidos que van más allá de aquellos derivados del uso de un solo método (Hamui-Sutton, 2013). En realidad, las formas de investigar pueden ser sui generis $\mathrm{y}$ totalmente idiosincráticas de investigador a investigador siguiendo el propósito de resolver el problema que ha definido.

R. Driver también ha insistido a lo largo de estos últimos años, reiteradamente, en la necesidad de ampliar el foco de atención de los cursos escolares de ciencias con objeto de hacerlos así más relevantes para los intereses del individuo y la sociedad moderna. 
Así, se han desarrollado programas de enseñanza que presentan los principios de la ciencia en los contextos que se consideran, en sí mismos, de uso práctico, que deberían reflejar mejor la propia actividad científica; de manera que los estudiantes, como futuros ciudadanos o científicos, lleguen a ser más conscientes de las posibilidades y limitaciones de la ciencia como una empresa humana. Esto se trata de tendencias en la concepción del currículo de ciencias cuya orientación viene determinada por el deseo de suministrar una educación liberal y por la importancia de que los jóvenes vean la relevancia de la ciencia para sus propias vidas; una perspectiva que se basa en nuestra comprensión del propio proceso de aprendizaje, en particular de cómo los jóvenes aprenden sobre su mundo físico (Driver, 1988)

La investigación científica se ha visto como un proceso mediante el cual se logra adquirir conocimientos relevantes y fidedignos sobre el mundo y el universo, y se incluye arbitrariamente en el a la investigación tecnológica. Ello con la utilización de un método sistemático llamado método científico cuyos procedimientos no se equiparan a este método, sino más al suigéneris de investigación tecnológica que tiene sus propias reglas.

Las etapas de la investigación científica pueden incluir la observación, la definición del problema, la logística (planificación, evaluación de las evidencias), la formulación de la hipótesis, la experimentación (prueba de la hipótesis), la evaluación y el análisis (https://www.lifeder.com/investigacioncientifica/).

Sin embargo, antes de plantear las etapas de la investigación científica es determinante tener en cuenta el objetivo general que se persigue, dado que esto condiciona los objetivos específicos a seguir y los métodos que se eligen para el logro de estos objetivos.

Todos conocemos que las enseñanzas de máster y doctorado tienen como finalidad que el estudiante adquiera una formación avanzada especializada en un ámbito científico, técnico o artístico o una formación en investigación.
En consecuencia, se trabajarán preferentemente las competencias de comprensión e integración de conocimientos, ampliación de la información, métodos de investigación, creatividad, innovación, diseño y desarrollo de investigaciones originales y comunicación de los avances tecnológicos, sociales y culturales tanto a públicos especializados como a los no especializados (Sanz de Acedo Lizarraga, 2016) sin embargo no hay mejor garantía de lograr esto que iniciar la formación de los estudiantes en investigación desde los grados inferiores, inclusive desde la educación básica.

La autora insiste en que el comentario que se ha realizado sobre esta competencia secunda la tesis de que son múltiples los factores que inciden en la decisión y de que es preciso estar alerta en el momento de tomarla, sobre todo cuando se trata de la realización personal y profesional. Aunque las decisiones difieren en el contenido y en la forma de llevarlas a cabo, la investigación ha descubierto una actuación básica y sistemática de la persona cuando decide $\mathrm{y}$ ha formulado unos principios generales psicológicos que caracterizan la conducta decisoria (Ibid. Pág. 102) e menciona que En general, la investigación demuestra que las competencias cognitivas se desarrollan cuando los alumnos movilizan o transfieren sus conocimientos y encuentran por sí mismos los recursos que les permitan resolver situaciones complejas, ya sea trabajando en ambientes enriquecidos con las Tecnologías de la Información y la Comunicación (TIC) o en ambientes tradicionales (Ibid. Pág. 116).

Aun así, otros autores han insistido en que el proceso científico es no lineal, impredecible y continuo. Kristy Wilson piensa que valuar la naturaleza de la ciencia es difícil con métodos que se basan en preguntas de opción múltiple o de escala Likert, así que evaluó el proceso científico utilizando "diagramas de flujo" en una metodología llamada Scientific Process Flowchart Assessment (SPFA) diseñada para evaluar la comprensión de los estudiantes del proceso científico. Los diagramas de flujo anteriores a posteriores mostraron una mejora estadísticamente significativa en el número de elementos y calificaciones para las dimensiones. 
La comparación de los términos utilizados y las conexiones entre los términos en los diagramas de flujo de los estudiantes reveló una comprensión mejorada y más matizada del proceso científico, especialmente en las áreas de aplicación a la sociedad y la comunicación dentro de la comunidad científica (Wilson \& Rigakos, 2016).

\section{Método}

La investigación es descriptiva y cuantitativa, que pretende elucidar procesos de investigación en jóvenes estudiantes que realizan proyectos de ingeniería o de ciencia. Las variables son las que se encuentran en los formatos aportados por el CONACYT y el COECyT de Coahuila, y evaluadas por jueces seleccionados por su prestigio estatal en el desarrollo de proyectos de investigación de los diversos campos. sus tipos, criterios para la selección de variables y su pertinencia.

El instrumento de investigación es un instrumento diseñado $\mathrm{y}$ aplicado por el CONACyT y el COECyT (ConsejoEstal de Ciencia y Tecnología), consta de un diseño diferente para el evaluación de los proyectos de ingeniería o de los de ciencias, con algunas variables que no se comparten en ambos. Las interrogantes se derivaron del marco de referencia de la investigación, medidos en un cuestionario tipo Likert, por lo cual se procedió a estandarizarlos para lograr la recodificación de ellas con la que fueron procesadas para obtener una escala centesimal y tratamientos más precisos de análisis multivariado, de acuerdo con el Manual de puntuación de la versión española del Cuestionario de Salud SF-36 de la Unitat de Recerca en Serveis Sanitaris Institut Municipal d'Investigació Mèdica (Investigació Mèdica, I. M. (1996).

Se obtuvieron 28 variables tipo Likert estandarizadas del instrumento de evaluación de Ingeniería, 8 de ellas categoriales 8 Variables complejas) y 28 ordinales en un instrumento previamente proporcionado por la FENACI, a través del Consejo Estatal de Ciencia y Tecnología de Coahuila. Estas variables fueron tratadas con estadística descriptiva, correlación por nivel educativo y Análisis Discriminante.
La muestra está constituida por 311 formatos de evaluación obtenidos de 135 proyectos, con los datos que generaron los 62 evaluadores de proyectos designados por el COECyT, dada su vasta experiencia como investigadores en Coahuila, que valoraron los proyectos de los estudiantes de forma independiente.Se esto se sigue que cada proyecto fue evaluado por al menos 3 investigadores con formatos preestablecidos en los rubros que se observan en la Tabla 1.

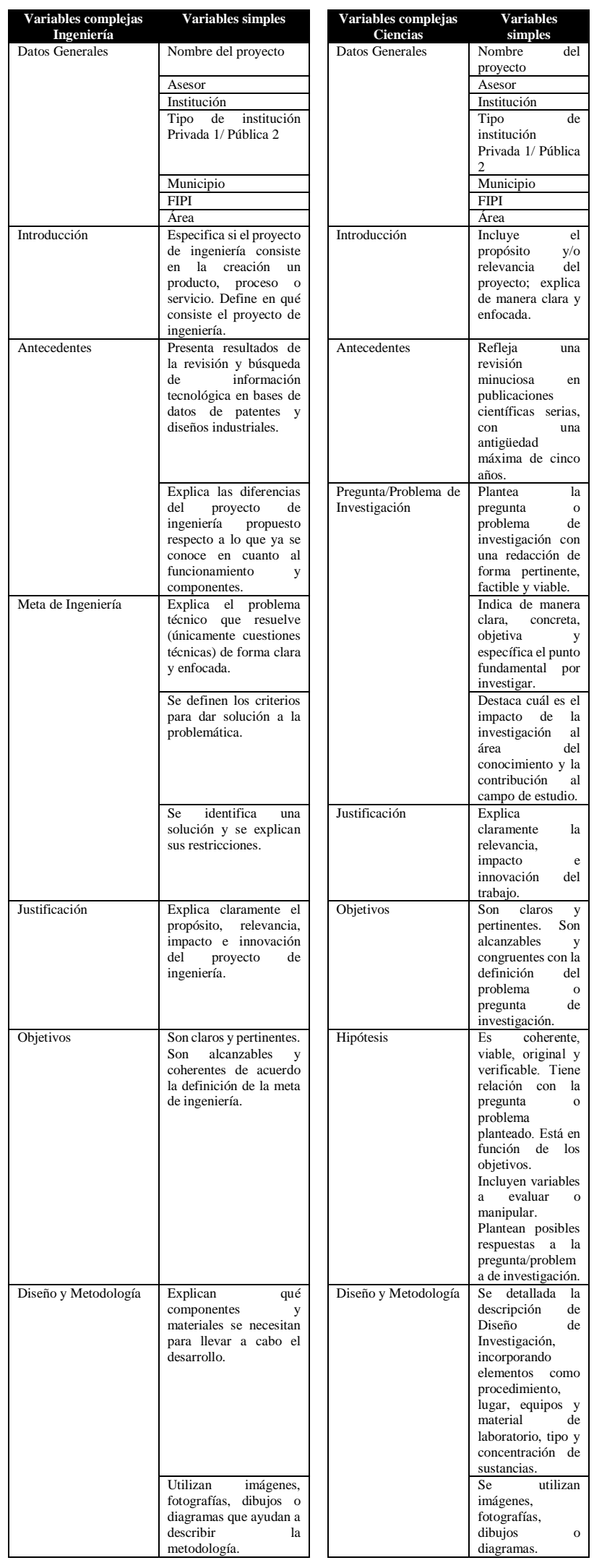

MUÑOZ-LÓPEZ, Temístocles, MARTÍNEZ-SOSA, Vanessa, RAMOSJAUBERT, Rocío Isabel y ESPERICUETA-MEDINA, Marta Nieves. El proceso de investigación de los estudiantes de la Feria Nacional de Ciencias e Ingeniería de Coahuila. Revista Ciencias de la Educación. 2020 

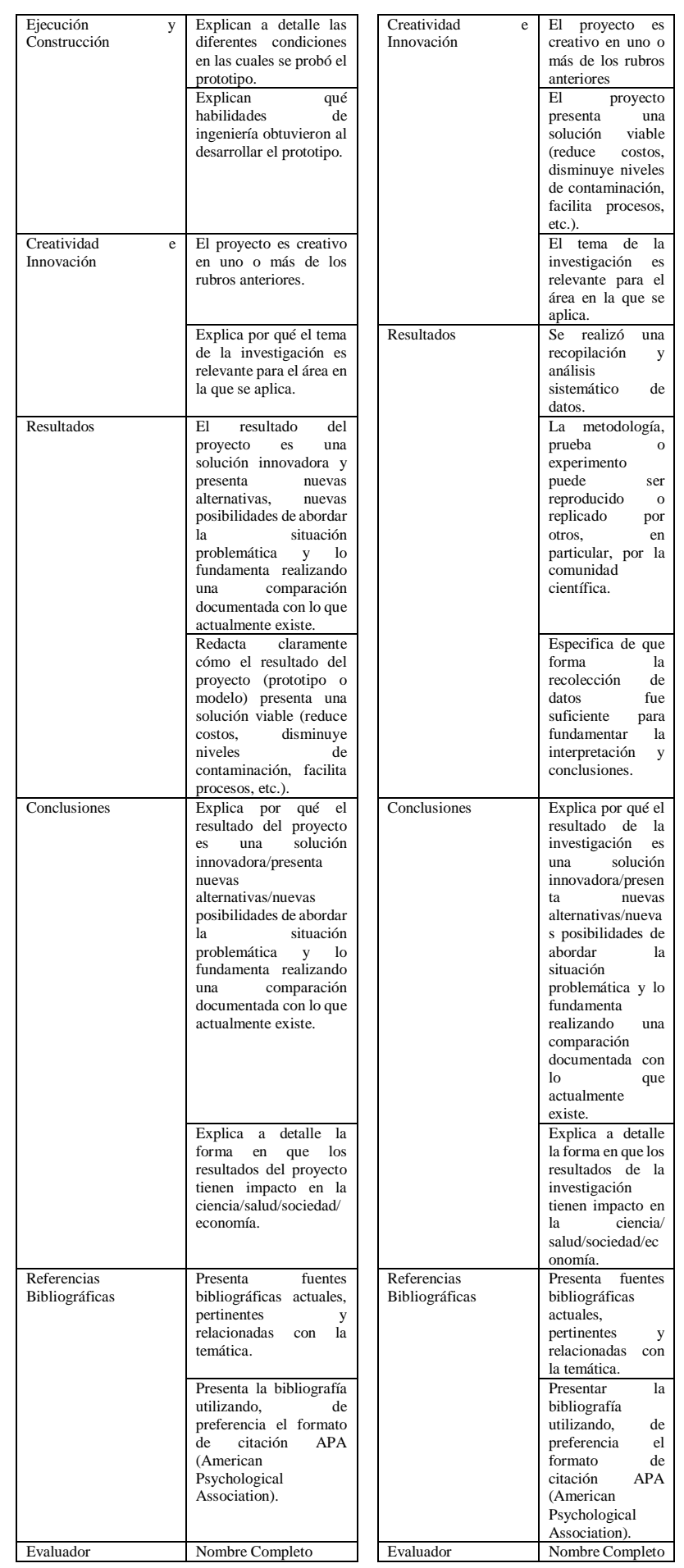

Tabla 1 Comparación de las Variables complejas y simples que participaron en el estudio están tomadas directamente de los formatos de evaluación de los proyectos, con las diferencias propias en algunos rubros dependiendo de si pertenecen a proyectos de ingeniería (izquierda) o de ciencias (derecha)

Con fines prácticos se decidió abreviar los nombres de las variables simples para su ingreso en los programas estadísticos y reducir la elaboración de las figuras en espacios más acordes a los requisitos de la publicación, por lo cual se utilizan las siguientes abreviaturas:
INT: Especifica si el proyecto de ingeniería consiste en la creación un producto, proceso o servicio. Define en qué consiste el proyecto de ingeniería.

ANTPresenta: Presenta resultados de la revisión y búsqueda de información tecnológica en bases de datos de patentes y diseños industriales.

ANTExplica: Explica las diferencias del proyecto de ingeniería propuesto respecto a lo que ya se conoce en cuanto al funcionamiento y componentes.

METAExplica: Explica el problema técnico que resuelve (únicamente cuestiones técnicas) de forma clara y enfocada.

METAcriterios: Se definen los criterios para dar solución a la problemática.

METAidentifsol: Se identifica una solución y se explican sus restricciones.

JUSTIFExplica: Explica claramente el propósito, relevancia, impacto e innovación del proyecto de ingeniería.

OBJ: Son claros y pertinentes. Son alcanzables y coherentes de acuerdo la definición de la meta de ingeniería.

DYMExplican: Explican qué componentes y materiales se necesitan para llevar a cabo el desarrollo.

DYMUtilizan: Utilizan imágenes, fotografías, dibujos o diagramas que ayudan a describir la metodología.

EYCExplcond: Explican a detalle las diferentes condiciones en las cuales se probó el prototipo.

EYCEhabiliding: Explican qué habilidades de ingeniería obtuvieron al desarrollar el prototipo.

CEIcreativo: El proyecto es creativo en uno o más de los rubros anteriores.

CEItemaREL: Explica por qué el tema de la investigación es relevante para el área en la que se aplica. 
RESinnov: El resultado del proyecto es una solución innovadora y presenta nuevas alternativas, nuevas posibilidades de abordar la situación problemática y lo fundamenta realizando una comparación documentada con lo que actualmente existe.

RESVIABLE: Redacta claramente cómo el resultado del proyecto (prototipo o modelo) presenta una solución viable (reduce costos, disminuye niveles de contaminación, facilita procesos, etc.).

CONCinnova: Explica por qué el resultado del proyecto es una solución innovadora/presenta nuevas alternativas/nuevas posibilidades de abordar la situación problemática y lo fundamenta realizando una comparación documentada con lo que actualmente existe.

CONCExplica: Explica a detalle la forma en que los resultados del proyecto tienen impacto en la ciencia/salud/sociedad/economía.

REFbiblio: Presenta fuentes
bibliográficas actuales, pertinentes y
relacionadas con la temática.

REFAPA: Presenta la bibliografía utilizando, de preferencia el formato de citación APA (American Psychological Association).

En la lectura de los resultados adicionalmente se escriben los nombres de las variables con texto en cursiva para evitar confusiones con el texto normal.

\section{Resultados}

\section{Estadística descriptiva}

Las variables que tuvieron los valores más altos (mayores a la Media más 1 Desviación estándar) corresponden a Especifica si el proyecto de ingeniería consiste en la creación un producto, proceso o servicio. Define en qué consiste el proyecto de ingeniería; Explica claramente el propósito, relevancia, impacto e innovación del proyecto de ingeniería; Explican qué componentes y materiales se necesitan para llevar a cabo el desarrollo y, Utilizan imágenes, fotografías, dibujos o diagramas que ayudan a describir la metodología.
En tanto que las variables con los valores más bajos son: Explican qué habilidades de ingeniería obtuvieron al desarrollar el prototipo y, la variable Presenta resultados de la revisión y búsqueda de información tecnológica en bases de datos de patentes y diseños industriales. Las demás variables se pueden considerar normales a una sigma (ver Tabla 2).

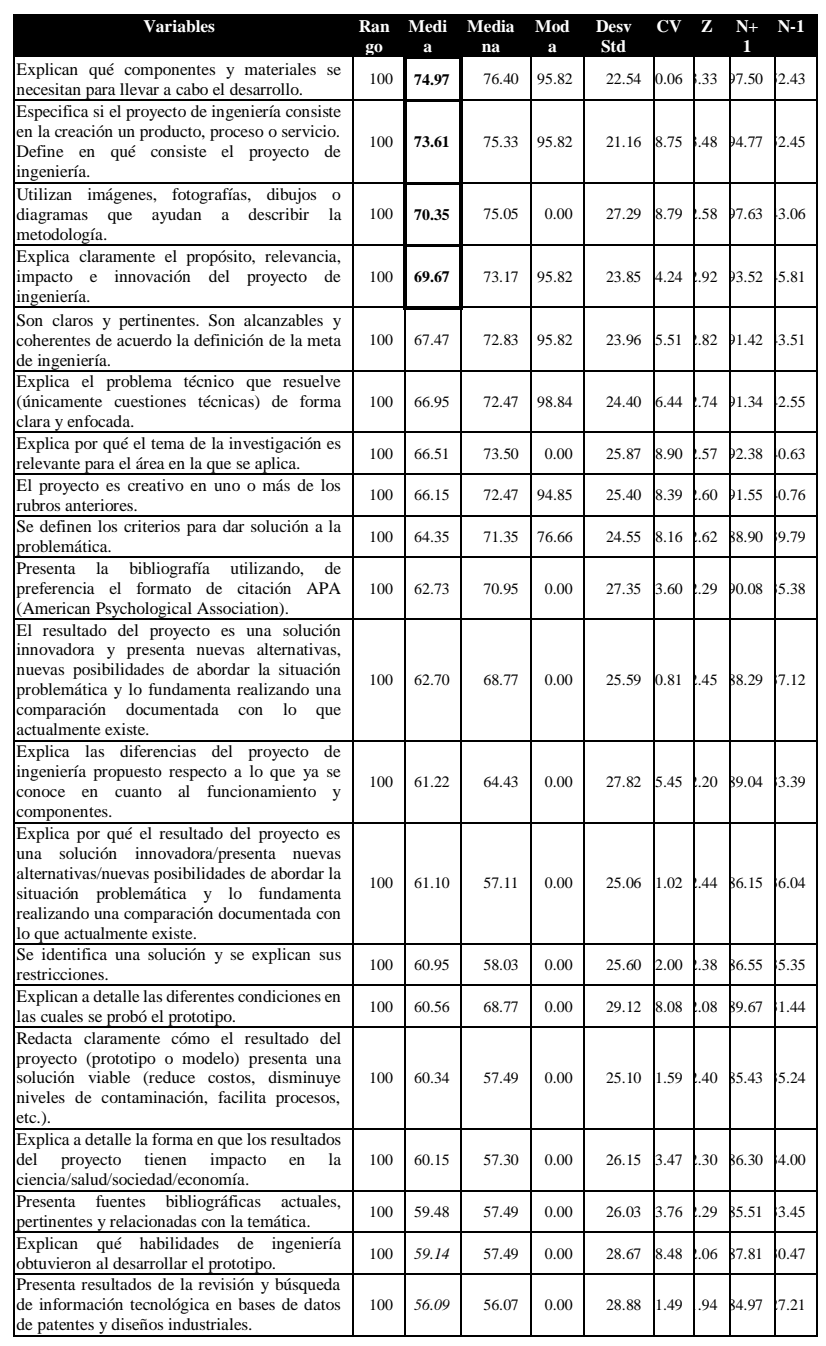

Tabla 2 Estadística descriptiva de los 311casos que participaron en el estudio, donde se muestra en casi todas una gran consistencia dado el valor $\mathrm{Z}$ mayor a 1.96, el promedio de las medias a una sigma denotadas por la Media de 64.22, la Desviación Estándar de 5.08, y la Media más una Desviación Estándar de 69.31 y menos una Desviación Estándar de 59.14 que permiten saber cuáles son las variables más y menos robustas

\section{Correlación}

Por otra parte, se hizo un tratamiento correlacional para denotar la asociación entre las variables de cada nivel educativo, con el propósito de mostrar el flujo de los procesos y clarificar cuáles las variables definen las secuencias metodológicas más relevantes entre los estudiantes al desarrollar sus proyectos tecnológicos. Para ello se utilizaron tablas y gráficas. 


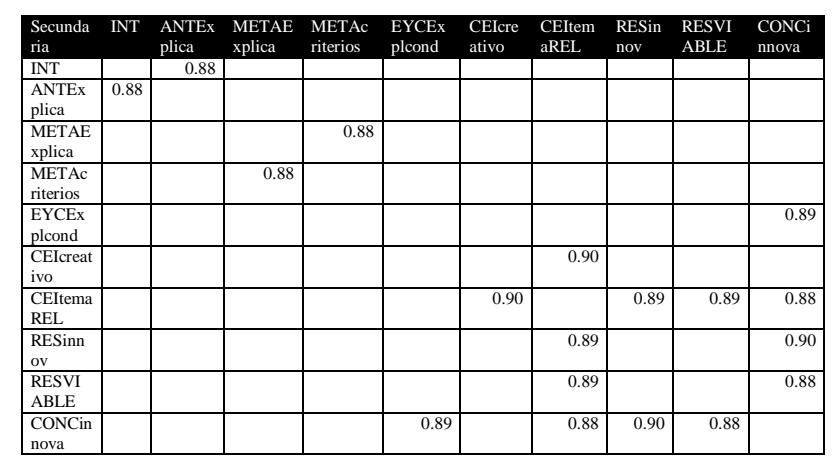

Tabla 3 Correlación en los proyectos del nivel de Secundaria con valores de corte sigmático $\geq 0.84$ y una $n=42$ para denotar las variables que intervienen en sus procesos de investigación

Donde intervienen con la Clave de las variables y su Descripción:

INT: Especifica si el proyecto de ingeniería consiste en la creación un producto, proceso o servicio. Define en qué consiste el proyecto de ingeniería.

ANTExplica: Explica las diferencias del proyecto de ingeniería propuesto respecto a lo que ya se conoce en cuanto al funcionamiento y componentes.

METAExplica: Explica el problema técnico que resuelve (únicamente cuestiones técnicas) de forma clara y enfocada.

METAcriterios: Se definen los criterios para dar solución a la problemática.

EYCExplcond: Explican a detalle las diferentes condiciones en las cuales se probó el prototipo.

CEIcreativo: El proyecto es creativo en uno o más de los rubros anteriores.

CEItemaREL: Explica por qué el tema de la investigación es relevante para el área en la que se aplica.

RESinnov: El resultado del proyecto es una solución innovadora y presenta nuevas alternativas, nuevas posibilidades de abordar la situación problemática y lo fundamenta realizando una comparación documentada con lo que actualmente existe.

RESVIABLE: Redacta claramente cómo el resultado del proyecto (prototipo o modelo) presenta una solución viable (reduce costos, disminuye niveles de contaminación, facilita procesos, etc.).
CONCinnova: Explica por qué el resultado del proyecto es una solución innovadora/presenta nuevas alternativas/nuevas posibilidades de abordar la situación problemática y lo fundamenta realizando una comparación documentada con lo que actualmente existe.

Graficadas las correlaciones observamos que la parte central de los procesos metodológicos de los estudiantes de secundaria esta nucleado en cuatro variables: Explica por qué el tema de la investigación es relevante para el área en la que se aplica; El resultado del proyecto es una solución innovadora y presenta nuevas alternativas, nuevas posibilidades de abordar la situación problemática y lo fundamenta realizando una comparación documentada con lo que actualmente existe; Redacta claramente cómo el resultado del proyecto (prototipo o modelo) presenta una solución viable (reduce costos, disminuye niveles de contaminación, facilita procesos, etc.) y Explica por qué el resultado del proyecto es una solución innovadora/presenta nuevas alternativas/nuevas posibilidades de abordar la situación problemática y lo fundamenta realizando una comparación documentada con lo que actualmente existe.

Por su parte Explica por qué el tema de la investigación es relevante para el área en la que se aplica es apoyado porque El proyecto es creativo en uno o más de los rubros anteriores y, cuando Explica por qué el resultado del proyecto es una solución innovadora/presenta nuevas alternativas/nuevas posibilidades de abordar la situación problemática y lo fundamenta realizando una comparación documentada con lo que actualmente existe, considerando que ésta variable de innovación es apoyada cuando Explican a detalle las diferentes condiciones en las cuales se probó el prototipo.

Quedan aisladas del núcleo central dos parejas sin relacionarse con las variables antes mencionadas: Especifica si el proyecto de ingeniería consiste en la creación un producto, proceso o servicio. Define en qué consiste el proyecto de ingeniería con la variable y Explica las diferencias del proyecto de ingeniería propuesto respecto a lo que ya se conoce en cuanto al funcionamiento y componentes y las otras dos variables asociadas. 
El otro par de variables asociadas fuera del núcleo central incluye que Explica el problema técnico que resuelve (únicamente cuestiones técnicas) de forma clara y enfocada y Se definen los criterios para dar solución a la problemática. Lo anterior se observa en la Figura 1.

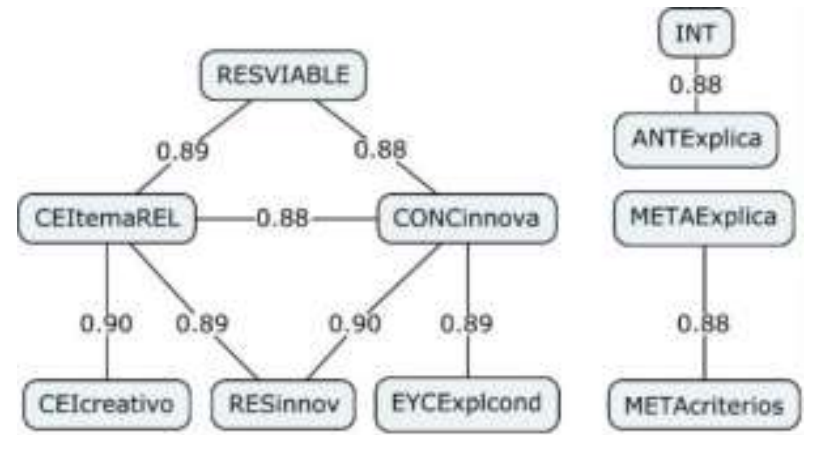

Figura 1 Constelación correlacional de la relación de variables de la metodología de investigación en proyectos del nivel de secundaria

En el caso de la Educación media superior resultan 15 variables correlacionadas significativamente con un corte sigmático con valores $\geq 0.75113$ casos.

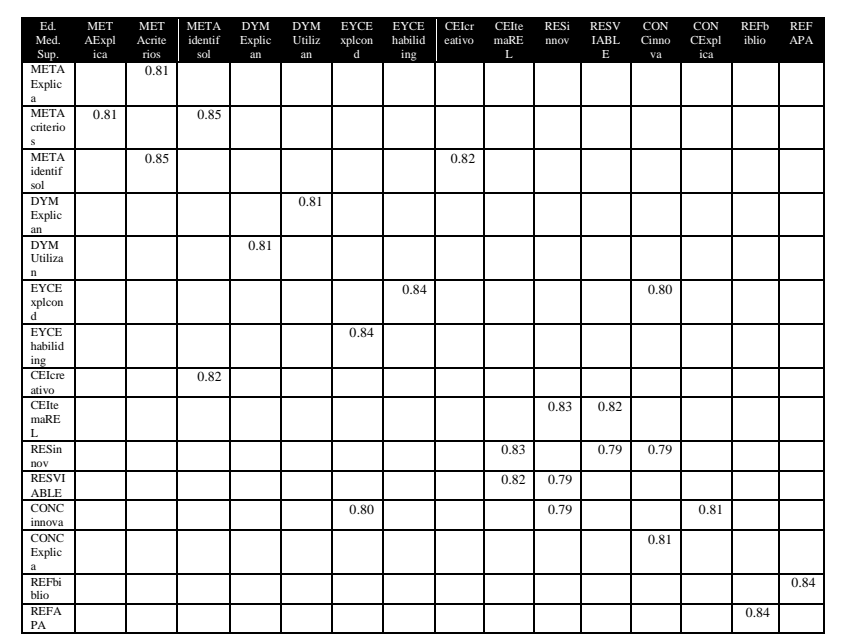

Tabla 4 Variables de investigación relacionadas en los proyectos de ingeniería de los estudiantes de educación media superior

\section{Donde}

METAExplica: Explica el problema técnico que resuelve (únicamente cuestiones técnicas) de forma clara y enfocada.

METAcriterios: Se definen los criterios para dar solución a la problemática.

METAidentifsol: Se identifica una solución y se explican sus restricciones.
DYMExplican: Explican qué componentes y materiales se necesitan para llevar a cabo el desarrollo.

DYMUtilizan: Utilizan imágenes, fotografías, dibujos o diagramas que ayudan a describir la metodología.

EYCExplcond: Explican a detalle las diferentes condiciones en las cuales se probó el prototipo.

EYCEhabiliding: Explican qué habilidades de ingeniería obtuvieron al desarrollar el prototipo.

CEIcreativo: El proyecto es creativo en uno o más de los rubros anteriores.

CEItemaREL: Explica por qué el tema de la investigación es relevante para el área en la que se aplica.

RESinnov: El resultado del proyecto es una solución innovadora y presenta nuevas alternativas, nuevas posibilidades de abordar la situación problemática y lo fundamenta realizando una comparación documentada con lo que actualmente existe.

RESVIABLE: Redacta claramente cómo el resultado del proyecto (prototipo o modelo) presenta una solución viable (reduce costos, disminuye niveles de contaminación, facilita procesos, etc.).

CONCinnova: Explica por qué el resultado del proyecto es una solución innovadora/presenta nuevas alternativas/nuevas posibilidades de abordar la situación problemática y lo fundamenta realizando una comparación documentada con lo que actualmente existe.

CONCExplica: Explica a detalle la forma en que los resultados del proyecto tienen impacto en la ciencia/salud/sociedad/economía.

REFbiblio: Presenta fuentes bibliográficas actuales, pertinentes y relacionadas con la temática.

REFAPA: Presenta la bibliografía utilizando, de preferencia el formato de citación APA (American Psychological Association). 
La figura que muestra la relación entre las variables de educación media superior también muestra un núcleo central constituido por siete variables interrelacionadas de acuerdo con el valor significativo mayor apuntó 75 , además hay tres núcleos aislados, uno de cuatro variables y dos de variables apareadas.

El núcleo central se articula alrededor de la variable El resultado del proyecto es una solución innovadora y presenta nuevas alternativas, nuevas posibilidades de abordar la situación problemática y lo fundamenta realizando una comparación documentada con lo que actualmente existe, y son ellas que Explica por qué el resultado del proyecto es una solución innovadora/presenta nuevas alternativas/nuevas posibilidades de abordar la situación problemática y lo fundamenta realizando una comparación documentada con lo que actualmente existe, que Explica por qué el tema de la investigación es relevante para el área en la que se aplica y que Redacta claramente cómo el resultado del proyecto (prototipo o modelo) presenta una solución viable (reduce costos, disminuye niveles de contaminación, facilita procesos, etc.).

A partir de la variable El resultado del proyecto es una solución innovadora y presenta nuevas alternativas, nuevas posibilidades de abordar la situación problemática y lo fundamenta realizando una comparación documentada con lo que actualmente existe, encontramos otra variable con gran peso, ya que articula una segunda parte del núcleo, y es porque Explica por qué el resultado del proyecto es una solución innovadora/presenta nuevas alternativas/nuevas posibilidades de abordar la situación problemática y lo fundamenta realizando una comparación documentada con lo que actualmente existe y de ella se asocia el hecho que Explica a detalle la forma en que los resultados del proyecto tienen impacto en la ciencia/salud/sociedad/economía.

Adicionalmente a esta variable que refiere a que el joven explica porque el proyecto es una innovación, se asocia a que Explican a detalle las diferentes condiciones en las cuales se probó el prototipo y ésta a que Explican qué habilidades de ingeniería obtuvieron al desarrollar el prototipo.
Ya fuera del núcleo central encontramos el agrupamiento de una secuencia formada por Explica el problema técnico que resuelve (únicamente cuestiones técnicas) de forma clara y enfocada, Se definen los criterios para dar solución a la problemática, Se identifica una solución y se explican sus restricciones y culmina finalmente en que El proyecto es creativo en uno o más de los rubros anteriores.

Los dos pares marginales al núcleo central se corresponden a su identidad con variables complejas.

Por una parte Explican qué componentes y materiales se necesitan para llevar a cabo el desarrollo, asociada con Utilizan imágenes, fotografías, dibujos o diagramas que ayudan a describir la metodología. Y en el otro par está que Presenta fuentes bibliográficas actuales, pertinentes y relacionadas con la temática asociada con que Presenta la bibliografía utilizando, de preferencia el formato de citación APA (American Psychological Association). Lo anterior se puede observar en las gráficas resultants de resultantes de la Figura 2.
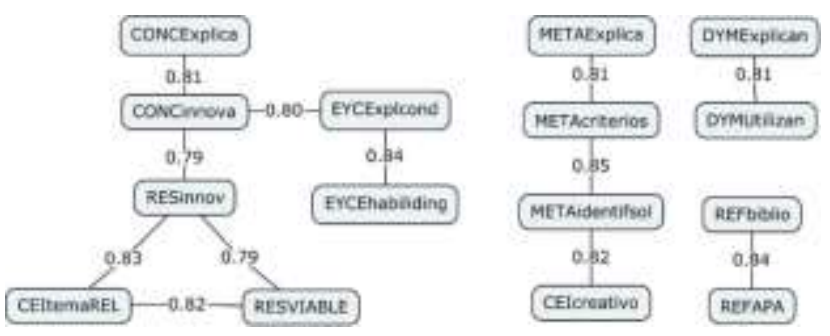

Figura 2 Constelación correlacional de la relación de variables de la metodología de investigación en proyectos del nivel de Educación Media Superior

En el caso del tercer nivel educativo que corresponde a la Educación Superior, con valores de corte sigmático $\geq 0.77$ y un total de 154 casos, vemos un patrón semejante de organización de variables. Un grupo central que nuclea mayor cantidad de variables, además de núcleos menores y pares aislados. 


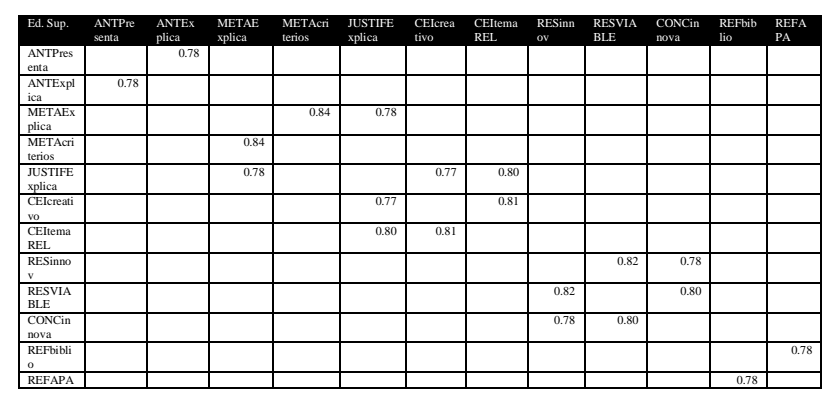

Tabla 5 Variables de investigación relacionadas en los proyectos de ingeniería de los estudiantes de Educación Superior

\section{Donde}

ANTPresenta: Presenta resultados de la revisión y búsqueda de información tecnológica en bases de datos de patentes y diseños industriales.

ANTExplica: Explica las diferencias del proyecto de ingeniería propuesto respecto a lo que ya se conoce en cuanto al funcionamiento y componentes.

METAExplica: Explica el problema técnico que resuelve (únicamente cuestiones técnicas) de forma clara y enfocada.

METAcriterios: Se definen los criterios para dar solución a la problemática.

JUSTIFExplica: Explica claramente el propósito, relevancia, impacto e innovación del proyecto de ingeniería.

CEIcreativo: El proyecto es creativo en uno o más de los rubros anteriores.

CEItemaREL: Explica por qué el tema de la investigación es relevante para el área en la que se aplica.

RESinnov: El resultado del proyecto es una solución innovadora y presenta nuevas alternativas, nuevas posibilidades de abordar la situación problemática y lo fundamenta realizando una comparación documentada con lo que actualmente existe.

RESVIABLE: Redacta claramente cómo el resultado del proyecto (prototipo o modelo) presenta una solución viable (reduce costos, disminuye niveles de contaminación, facilita procesos, etc.).
CONCinnova: Explica por qué el resultado del proyecto es una solución innovadora/presenta nuevas alternativas/nuevas posibilidades de abordar la situación problemática y lo fundamenta realizando una comparación documentada con lo que actualmente existe.

REFbiblio: Presenta fuentes bibliográficas actuales, pertinentes y relacionadas con la temática.

REFAPA: Presenta la bibliografía utilizando, de preferencia el formato de citación APA (American Psychological Association).

En el caso de educación superior también observamos un núcleo central de cinco variables interrelacionadas, así como un pequeño núcleo de 3 variables aisladas, así como dos padres de variables también aisladas.

El núcleo central se caracteriza por tener como eje la variable Explica claramente el propósito, relevancia, impacto e innovación del proyecto de ingeniería, y en ella se articulan la variable El proyecto es creativo en uno o más de los rubros anteriores y la variable Explica por qué el tema de la investigación es relevante para el área en la que se aplica, ambas también están unidas entre sí. Adicionalmente tiene otra variable denominada $\mathrm{Se}$ definen los criterios para dar solución a la problemática, que a su vez se asocia a Explica el problema técnico que resuelve (únicamente cuestiones técnicas) de forma clara y enfocada.

El núcleo menor de tres variables integra la asociación de El resultado del proyecto es una solución innovadora y presenta nuevas alternativas, nuevas posibilidades de abordar la situación problemática y lo fundamenta realizando una comparación documentada con lo que actualmente existe; Redacta claramente cómo el resultado del proyecto (prototipo o modelo) presenta una solución viable (reduce costos, disminuye niveles de contaminación, facilita procesos, etc.) y Explica por qué el resultado del proyecto es una solución innovadora/presenta nuevas alternativas/nuevas posibilidades de abordar la situación problemática y lo fundamenta realizando una comparación documentada con lo que actualmente existe. 
Finalmente, los pares aislados que aparecen son, el primero Presenta resultados de la revisión y búsqueda de información tecnológica en bases de datos de patentes y diseños industriales y Explica las diferencias del proyecto de ingeniería propuesto respecto a lo que ya se conoce en cuanto al funcionamiento y componentes y el segundo par, Presenta fuentes bibliográficas actuales, pertinentes y relacionadas con la temática con la variable Presenta la bibliografía utilizando, de preferencia el formato de citación APA (American Psychological Association). Lo anteriormente explicado de Educación Suprior se muestra en la Figura 3.

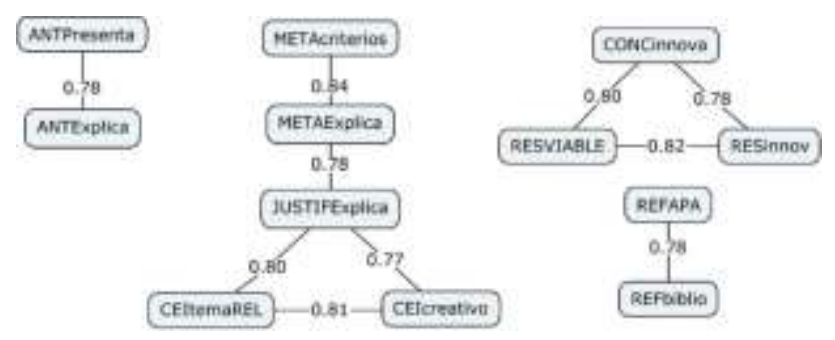

Figura 3 Constelación correlacional de la asociación de variables de la metodología de investigación en proyectos del nivel de Educación Superior

\section{Concordancias y diferencias de asociación}

Hay dos posibilidades de establecer las concordancias y diferencias el proceso de investigación que siguen los estudiantes para proponer sus proyectos de ingeniería, en el primer caso podemos analizar las estructuras de las variables asociadas y sus implicaciones, y en un segundo término las variables comunes que constituyen esas estructuras por nivel educativo. Encontramos que las variables que constituyen el núcleo central de educación secundaria se repiten también en los proyectos presentados en el nivel de preparatoria y en la educación superior, y estas son que explica por qué el tema de la investigación es relevante para el área en la que se aplica, que el resultado del proyecto es una solución innovadora y presenta nuevas alternativas, nuevas posibilidades de abordar la situación problemática y lo fundamenta realizando una comparación documentada con lo que actualmente existe, que redacta claramente cómo el resultado del proyecto (prototipo o modelo) presenta una solución viable (reduce costos, disminuye niveles de contaminación, facilita procesos, etc.)
Y que explica por qué el resultado del proyecto es una solución innovadora/presenta nuevas alternativas/nuevas posibilidades de abordar la situación problemática y lo fundamenta realizando una comparación documentada con lo que actualmente existe. Además, comparte con los otros niveles que explica por qué el tema de la investigación es relevante para el área en la que se aplica.

Se observa que solo en secundaria el estudiante especifica si el proyecto de ingeniería consiste en la creación un producto, proceso o servicio y define en qué consiste el proyecto. Solamente en preparatoria se presentas las variables donde se explican a detalle las diferentes condiciones en las cuales se probó el prototipo, explican qué habilidades de ingeniería obtuvieron al desarrollar el prototipo, se identifica una solución y se explican sus restricciones, se explican qué componentes y materiales se necesitan para llevar a cabo el desarrollo y utilizan imágenes, fotografías, dibujos o diagramas que ayudan a describir la metodología.

Solamente en Educación Superior se integran las variables que indican que presenta resultados de la revisión y búsqueda de información tecnológica en bases de datos de patentes y diseños industriales, así como la variable que mide si explica claramente el propósito, relevancia, impacto e innovación del proyecto de ingeniería.

\section{Análisis discriminante}

El análisis discriminante nos muestra la identidad de un tipo de proyecto con respecto a los demás, de acuerdo a los niveles educativos participantes en la Feria Nacional de la ciencia y la investigación. En este caso se muestra que la educación superior tiene una identidad del $49.68 \%$ en sus propios proyectos desde la perspectiva metodológica, así como el $27.74 \%$ con proyectos de preparatoria y el $22.58 \%$ con los de Secundaria, considerando 155 casos. para el caso de preparatoria encontramos que guardan una identidad de 50 por ciento entre ellos mismos con 114 casos, y en los de Secundaria este porcentaje de identidad es del $52.38 \%$ en un total de 42 casos. Lo anterior se expresa mejor en la Tabla 6. 


\begin{tabular}{|l|r|r|r|rr|}
\hline \multicolumn{5}{|c|}{ Predicción \% } \\
\hline Actual & $\begin{array}{l}\text { Ed. } \\
\text { Superior }\end{array}$ & Preparatoria & Secundaria & $\begin{array}{l}\text { Total } \\
\text { casos }\end{array}$ & de \\
\hline Ed. Superior & 49.68 & 27.74 & 22.58 & 155 \\
\hline Preparatoria & 28.95 & 50.00 & 21.05 & 114 \\
\hline Secundaria & 33.33 & 14.29 & 52.38 & 42 \\
\hline Total & & & & 311 \\
\hline
\end{tabular}

Tabla 6 Análisis discriminante de las 311 evaluaciones de proyectos de tres niveles educativos presentados en la FENACI de Coahuila

\section{Conclusiones}

Los proyectos de ingeniería presentados y la Feria Nacional de Ciencias realizada en el estado de Coahuila, tienen particularidades distintas en cada nivel educativo, aunque conservan una identidad propia al menos en el $50 \%$ de acuerdo con el análisis discriminante. Para el caso de educación básica se observó que su núcleo central y un par aislado tienen en común variables que se repiten en los otros niveles educativos.

Respecto a la metodología utilizada en este análisis habrá que puntualizar que los formatos que utilizaron los evaluadores estaban en escalas Likert $y$ fueron convertidos a puntuaciones estandarizadas para permitir un análisis multivariado o más detallado que solamente podría realizarse si tuviésemos los datos más espaciados en una escala centesimal, dado que la escala tipo Likert solamente permite el análisis de frecuencias y porcentajes o algún otro tipo análisis no paramétrico que tienen menor precisión.

A lo largo de la comparación pudimos observar siempre la presencia de un núcleo central de variables que caracterizan cada nivel educativo estudiado, así como subgrupos aislados de este núcleo central con variables que resultan relevantes para ese nivel, qué sin embargo no se encuentran directamente ligados a la explicación central. Lo anterior no quiere decir que las variables no pertenecen al modelo, más bien qué esas variables son parte del modelo global los proyectos presentados en ese nivel, pero no se encuentran articulados al núcleo central en todos los casos analizados y evaluados por los distintos jueces.
Con la evidencia empírica de la información obtenida en este estudio, podemos afirmar que la investigación para desarrollar productos de ingeniería dentro de la feria nacional mediante la elaboración de proyectos estudiantiles tiene procesos originales que no se sujetan a una metodología estricta, antes bien, los estudiantes desarrollan sus proyectos con ayuda de los docentes proponiendo y desarrollando soluciones paso a paso, en cada etapa de la investigación.

Y esto no es del todo cierto cuando se les pide llenar los requisitos protocolarios de los formatos, que, en todo caso, son requerimientos burocráticos del concurso. en este contexto también podemos afirmar que los proyectos tienen una identidad metodológica en promedio del $50 \%$ de acuerdo con el nivel educativo donde se presentan a concursar, de acuerdo con el análisis discriminante.

\section{Referencias}

COECyT. (2018). Feria de Ciencias y Creatividad 2018 - Consejo Estatal de Ciencia y Tecnología. Convocatoria. https://coecytcoahuila.gob.mx/feria-cienciascreatividad-2018/

Driver, R. (1988). Investigación y experiencias didácticas. Un enfoque constructivista para el desarrollo del currículo en ciencias. Enseñanza de Las Ciencias, 6(2), 109-120. Retrieved from https://ddd.uab.cat/pub/edlc/02124521v6n2/021 24521v6n2p109.pdf

Flores-Camacho, F. (2012). La enseñanza de la ciencia en la educación básica en México (I. N. Investigación Educativa, ed.). Retrieved from http://www.inee.edu.mx

Hamui-Sutton, A. (2013). Un acercamiento a los métodos mixtos de investigación en educación médica. Investigación En Educación Médica, 2(8), 211-216. https://doi.org/10.1016/s20075057(13)72714-5

Hueso, A., \& Cascant, M. J. (2012). Metodología y Técnicas Cuantitativas de Investigación (Universitat Politécnica de Valencia, ed.). Retrieved from www.lalibreria.upv.es 
Sanz de Acedo Lizarraga, M. L. (2016). Competencias cognitivas en Educación Superior (Primera; N. de E. S.A., ed.). Madrid: Ed. Narcea.

Wilson, K. J., \& Rigakos, B. (2016). Department of Biology. CBE—Life Sciences Education, (2), 14. https://doi.org/10.1187/cbe.15-10-0212 